Check for updates

Cite this: Chem. Sci., 2018, 9, 5781

\title{
Radical difluoromethylthiolation of aromatics enabled by visible light $\uparrow$
}

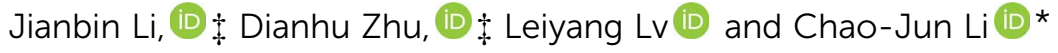 \\ Direct introduction of a difluoromethylthio group $\left(-\mathrm{SCF}_{2} \mathrm{H}\right)$ to arenes represents an efficient route to access \\ a valuable catalogue of organofluorines; however, to realize this transformation under metal-free and mild \\ conditions still remains challenging and rarely reported. Herein, a metal-catalyst-free and redox-neutral \\ innate difluoromethylthiolation method with a shelf-stable and readily available reagent, $\mathrm{PhSO}_{2} \mathrm{SCF}_{2} \mathrm{H}$, \\ under visible light irradiation is described. This light-mediated protocol successfully converts a broad \\ spectrum of arenes and heteroarenes to difluoromethylthioethers in the absence of noble metals and \\ stoichiometric amounts of additives.
}

Received 12th April 2018

Accepted 28th May 2018

DOI: $10.1039 / \mathrm{c} 8 \mathrm{sc} 01669 \mathrm{k}$

rsc.li/chemical-science

attack of an appropriate thiolate $\left(\mathrm{RS}^{-}\right)$to some " $\mathrm{CF}_{2}$ " species, ${ }^{9}$ typically a difluoromethyl carbene (:CF $),{ }^{10}$ (Scheme 1a, left). A complementary but less common approach to assemble $-\mathrm{SCF}_{2} \mathrm{H}$ is difluoromethylation of disulfides using nucleophilic difluoromethyl sources (e.g., activated $\left.\mathrm{TMSCF}_{2} \mathrm{H}\right) .{ }^{11} \mathrm{~A}$ major step forward to expand the substrate scope was made by the Gooßen group who described a stepwise synthetic route involving preformed thiocyanates and the subsequent copper-mediated Langlois type nucleophilic substitution by $\mathrm{TMSCF}_{2} \mathrm{H}$ (Scheme 1a, right). ${ }^{3 a, 12}$ Nevertheless, these indirect methods still suffer from a limited substrate scope. In addition, they usually necessitate strong bases, harsh thermal conditions and environmentally unfriendly reagents to generate reactive thiolates and " $\mathrm{CF}_{2}$ " species.

To address these issues, a key contribution was made by Shen and his co-workers who delineated the first nucleophilic difluoromethylthiolating reagent 1, [(SIPr) $\left.\mathrm{Ag}\left(\mathrm{SCF}_{2} \mathrm{H}\right)\right]$ (Scheme 1b). ${ }^{13}$ In the presence of transition metals $(\mathrm{M}=\mathrm{Pd}, \mathrm{Cu})$, this complex could couple with diverse aryl and heteroaryl halides, invention detailed that the $\mathrm{C}-4$ position bearing $-\mathrm{SCF}_{2} \mathrm{H}$ was identified as the most preferable structure. Furthermore, the important role of $-\mathrm{SCF}_{2} \mathrm{H}$ in pharmaceuticals and agrochemicals is evidenced by its frequent enrolment in other bioactive compounds, e.g., herbicide SSH-108, ${ }^{5}$ nifedipine analogue, ${ }^{6}$ and thymol analogue ${ }^{7}$ (Fig. 1a).

Despite the intriguing pharmaceutical potential exhibited by difluoromethylthioethers, their widespread application remains limited possibly owing to a lack of efficient preparative methods. ${ }^{8}$ Classical and commonly used approaches to synthesize difluoromethylthioethers employ the nucleophilic

Department of Chemistry, McGill University, 801 Sherbrooke Street West, Montreal, QC H3A 0B8, Canada.E-mail: cj.li@mcgill.ca

$\dagger$ Electronic supplementary information (ESI) available. See DOI: 10.1039/c8sc01669k

\$ These authors contributed equally to this work.

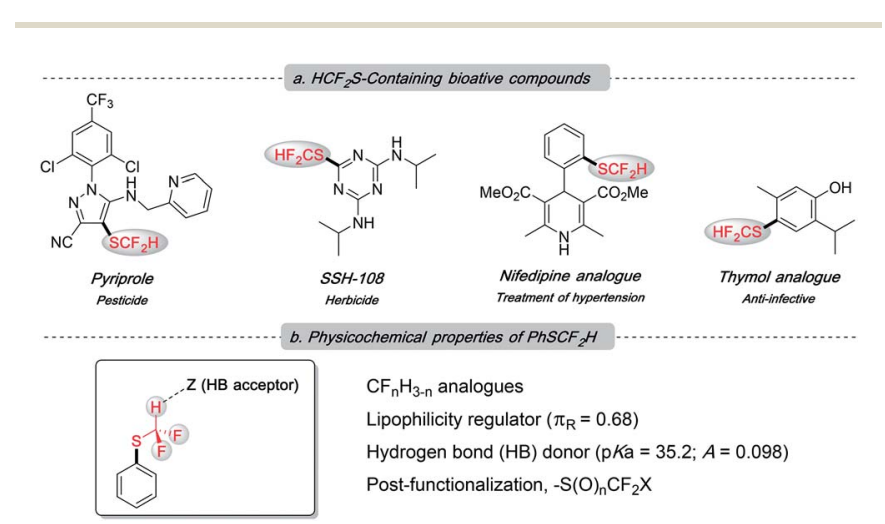

Fig. 1 (a) Frequent appearance of the $-\mathrm{SCF}_{2} \mathrm{H}$ residue in bioactive molecules; (b) overview of the uniqueness of $-\mathrm{SCF}_{2} \mathrm{H}$. 


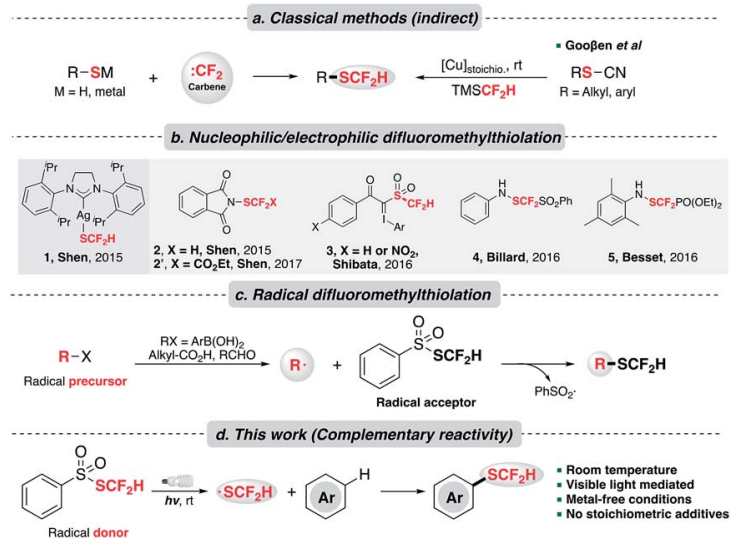

Scheme 1 Different recipes to install $-\mathrm{SCF}_{2} \mathrm{H}$ on arenes. (a) Classical methods to prepare difluoromethylthioethers; (b) direct difluoromethylthiolating reagents (1: nucleophilic; 2-5: electrophilic); (c) radical difluoromethylthiolation wherein $\mathrm{PhSO}_{2} \mathrm{SCF}_{2} \mathrm{H}$ acts as a radical acceptor; (d) this work: catalyst-free and redox-neutral innate difluoromethylthiolation with $\mathrm{PhSO}_{2} \mathrm{SCF}_{2} \mathrm{H}$ as the radical source under visible light.

triflates and diazonium compounds to prepare difluoromethylthioethers. Complementarily, the same group debuted an electrophilic difluoromethylthiolating reagent $2,^{\mathbf{1 4}}$ which could undergo a Friedel-Craft type $\mathrm{C}_{\mathrm{sp}^{2}}-\mathrm{H}$ difluoromethylthiolation on various $\mathrm{N}$-heteroarenes. Shortly after, Shibata et al. uncovered a hypervalent difluoromethanesulfonyliodonium ylide reagent 3 , which efficiently difluoromethylthiolated $\mathrm{N}$ heterocycles under copper catalysis. ${ }^{15}$ Moreover, the Billard, ${ }^{16}$ Besset $^{17}$ and Shen $^{18}$ groups independently synthesized three electrophilic $-\mathrm{SCF}_{2} \mathrm{FG}$ group transfer reagents $\left(\mathrm{FG}=\mathrm{PhSO}_{2}\right.$, $\mathrm{PO}(\mathrm{OEt})_{2}$ and $\mathrm{CO}_{2}$ Et respectively), although a separate reductive workup was necessary to give difluoromethylthioethers. Inspired by these elegant examples, other difluoromethylthiolating systems were unveiled. ${ }^{7,19}$

Alternatively, difluoromethylthiolation could be operated in a radical pathway. $S$-(Difluoromethyl)benzenesulfonothioate, $\mathrm{PhSO}_{2} \mathrm{SCF}_{2} \mathrm{H}$, invented in 2016 by Shen et al., was revealed as an effective difluoromethylthiolating reagent (Scheme 1c). ${ }^{20}$ Mechanistically, it was proposed to execute as a radical acceptor and combine with the alkyl or aryl radicals generated from the Ag/persulfate-involved oxidation event. This reactivity was further elaborated in two recent studies on the ring-opening difluoromethylthiolation of cycloalkanols ${ }^{21}$ and the preparation of difluoromethylthioesters by using aldehydes as acyl radical precursors. $^{22}$

Promising though these strategies are, the main drawbacks lie in their requirement of relatively high thermal energy (ranging from 50 to $120{ }^{\circ} \mathrm{C}$ ), or involvement of precious metal catalysts and stoichiometric amounts of additives. In the case of $-\mathrm{SCF}_{2} \mathrm{FG}$ group transfer, additional steps were required to furnish the final thioether products. Clearly, a more sustainable synthetic protocol that features mild conditions and step economy is highly desired.

In 2017, our group disclosed a radical trifluoromethylation reaction on arenes with a novel sulfone reagent 6 (Scheme 2a). ${ }^{23}$

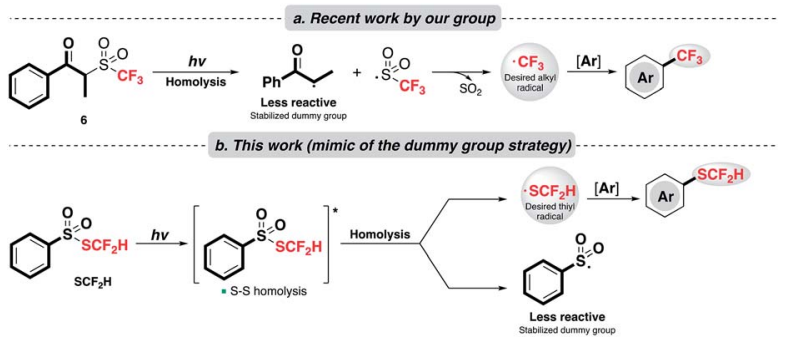

Scheme 2 (a) Controlled radical generation via the "dummy fragment" concept; (b) this work: catalyst-free and redox-neutral innate difluoromethylthiolation enabled by light.

With light irradiation, 6 engenders two twin radicals via Norrish type I cleavage as the sulfinyl radical fragments further to produce the $\mathrm{CF}_{3}$ radical and implements trifluoromethylation. The success of this chemistry lies in the judicious design of the reagent structure. In the so-called "dummy group" strategy, the undesirable radical produced by homolysis of $\mathbf{6}$ is diminished in reactivity by the "captodative effect" ${ }^{24}$ as well as sterics.

Inspired by this work, we would like to apply a similar strategy and achieve a rarely known direct radical difluoromethylthiolation (Scheme 2b). A key component of this project is to explore and identify a suitable $\mathrm{SCF}_{2} \mathrm{H}$ radical precursor. After a careful examination of the literature, we consider that the thiosulfonate reagent, $\mathrm{PhSO}_{2} \mathrm{SCF}_{2} \mathrm{H}$, might be applicable, although it was documented to be a radical acceptor as its principle utility. ${ }^{20,22}$ We proposed that the homolytic fragmentation of $\mathrm{PhSO}_{2} \mathrm{SCF}_{2} \mathrm{H}$ with a suitable light source is feasible due to the well-studied propensity of homolysis of the S-S bond ${ }^{25}$ and the stabilization of the resulting sulfonyl radical exerted by the resonance effect of the phenyl group. ${ }^{26}$ The stabilization of the dummy radical is beneficial in two ways: (1) it facilitates homolytic bond scission and allows the accumulation of $\mathrm{SCF}_{2} \mathrm{H}$ radicals; (2) it is expected to cause less competition toward the targeted reaction pathway. Radical addition of the desired $\mathrm{SCF}_{2} \mathrm{H}$ radical will furnish the target product.

As a part of our interest in photo-induced fluorine chemistry, we wish to document a photo-induced metal-free aromatic difluoromethylthiolation protocol with $\mathrm{PhSO}_{2} \mathrm{SCF}_{2} \mathrm{H}$, wherein $\mathrm{PhSO}_{2} \mathrm{SCF}_{2} \mathrm{H}$ was utilized as a difluoromethylthiyl radical source. To the best of our knowledge, direct aromatic difluoromethylthiolation realized by radical attack of $\mathrm{SCF}_{2} \mathrm{H}$ radical on arenes remained unexplored.

To commence the study, $N$-methylindole 1a was chosen as a model substrate and treated with difluoromethylthiolating sources in $\mathrm{CH}_{3} \mathrm{CN}$ under an inert atmosphere based on some literature parallels on difluoromethylthiolation (see $\mathrm{ESI} \dagger$ for full details). Although no desired product was detected when using $\mathrm{BnSCF}_{2} \mathrm{H}$, the reaction with $\mathrm{PhSO}_{2} \mathrm{SCF}_{2} \mathrm{H}$ proceeded as expected and upon 16 hours of UV irradiation, 20\% yield of the desired product was obtained (Table 1, entries 1 and 2). Using compact fluorescence lamps (CFL) as a light source gave 64\% yield of the target compound (entry 3 ). Fortunately, prolonging the reaction time enabled the consumption of unreacted substrates and increased the yield to $80 \%$ (entry 4). The 
Table 1 Selected results of evaluation under various conditions ${ }^{a}$

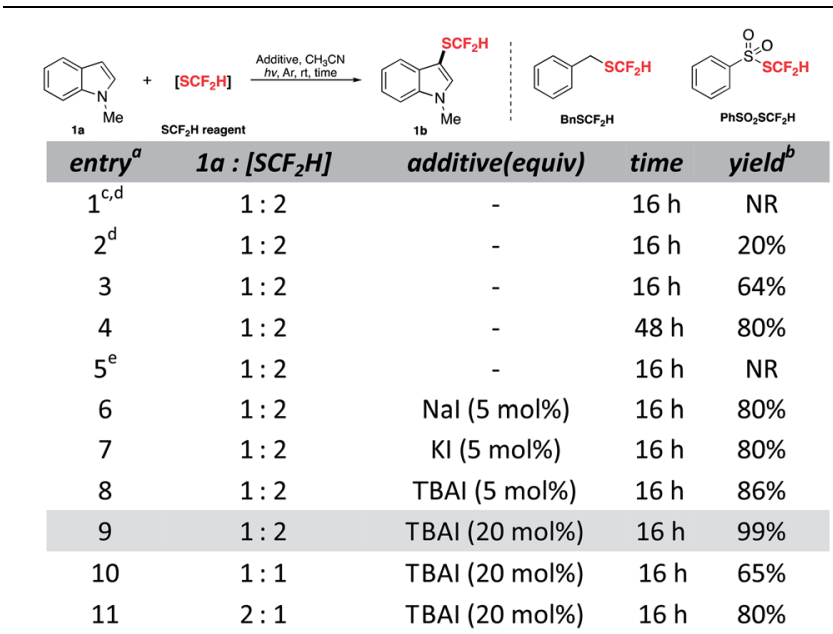

${ }^{a}$ Abbreviations: CFL, compact fluorescent lamp; rt, room temperature; TBAI, tetrabutylammonium iodide; NR, no reaction. ${ }^{b}$ All reactions were conducted with $0.10 \mathrm{mmol} \mathrm{1a}, 0.20 \mathrm{mmol} \mathrm{PhSO}_{2} \mathrm{SCF}_{2} \mathrm{H}, 0.020 \mathrm{mmol}$ TBAI in $1.0 \mathrm{~mL} \mathrm{CH}_{3} \mathrm{CN}$ under argon with irradiation of two $40 \mathrm{~W}$ CFL unless otherwise noted. ${ }^{c}$ The yield was determined by ${ }^{1} \mathrm{H}$ NMR analysis using 1,3,5-trimethoxybenzene as the internal standard. ${ }^{d} \mathrm{BnSCF}_{2} \mathrm{H}$ as the difluoromethylthiolating source. ${ }^{e}$ Six $254 \mathrm{~nm} 2.5 \mathrm{~W}$ UV lamps (photo-box). ${ }^{f}$ In the dark. essential role played by light was illustrated by the control experiment as the dark condition disabled the reaction completely (entry 5 and see ESI $\dagger$ for details on control experiments). Recent work by our group revealed the unique properties of NaI, e.g., high reducing ability along with low nucleophilicity, ${ }^{27}$ which were helpful in radical generation. Therefore, we expected the $\mathrm{SCF}_{2} \mathrm{H}$ radical generation would be accelerated by a complementary reductive pathway. Gratifyingly, catalytic incubation of iodide gave similarly good yield in a shortened reaction time (entries 6-8). Among the tested iodides, tetrabutylammonium iodide (TBAI) offered the highest yield (entry 8). Further increment of TBAI loading could promote the reaction to be quantitative (entry 9). Conforming to other electrophilic difluoromethylthiolation studies, 1a as a limiting reagent would be more profitable as an excess of difluoromethylthiolating reagent is crucial to maintain a decent level of active difluoromethylthiolating species (entries 10 and 11).

With the optimal conditions identified, the generality of this method was examined (Scheme 3). Initially, the functional group tolerance of different indoles was investigated. In general, indoles bearing substituents with different electronic and steric properties at various sites are compatible with the optimal conditions. Reaction rates of substrates with electrondonating groups were higher than those with electron-withdrawing groups. Satisfactorily, quantitative yield was obtained for non-substituted indole (2b). Product formation was not

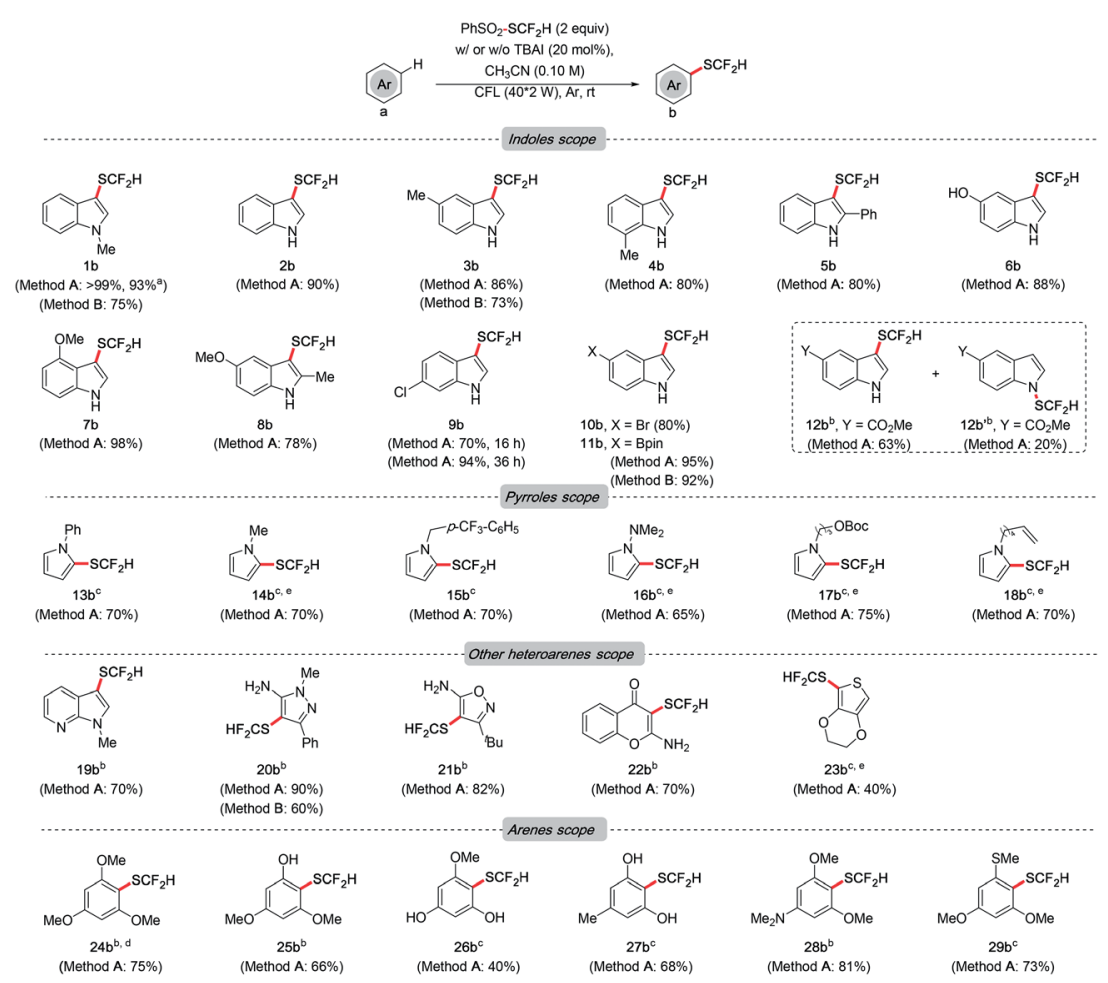

Scheme 3 Scope of arenes. Method A: arene $(0.10 \mathrm{mmol}), \mathrm{PhSO}_{2} \mathrm{SCF}_{2} \mathrm{H}(0.20 \mathrm{mmol})$, TBAl $(0.020 \mathrm{mmol})$ in $1.0 \mathrm{~mL} \mathrm{CH} \mathrm{CN}_{3}$ under argon for CFL irradiation at rt for $16 \mathrm{~h}$. Method $\mathrm{B}$ : arene $(0.10 \mathrm{mmol}), \mathrm{PhSO}_{2} \mathrm{SCF}_{2} \mathrm{H}(0.20 \mathrm{mmol})$ in $1.0 \mathrm{~mL} \mathrm{CH} \mathrm{CNN}_{3}$ under argon for CFL irradiation at rt for $48 \mathrm{~h}$. The yields in the parentheses refer to the isolated ones unless otherwise specified. Volatility resulted in the low isolated yield of $17 \mathrm{~b}$ and $18 \mathrm{~b}$. ${ }^{a}$ Reaction performed on the $0.40 \mathrm{mmol}$ scale. ${ }^{b}$ The reactions were performed for $24 \mathrm{~h}$. ${ }^{c}$ The reactions were performed for $48 \mathrm{~h}$. ${ }^{d} 4$ equiv. $\mathrm{PhSO}_{2} \mathrm{SCF}_{2} \mathrm{H}$ were used. ${ }^{e}$ Yields are quantified by GC-MS due to the volatility of target compounds. 
affected by the methyl group at the C-5 or C-7 position ( $3 \mathbf{b}$ and 4b). Even in the presence of a phenyl group at the C-2 position, a decent yield can also be achieved (5b). Notably, the reaction proceeded smoothly in indole tethering a phenolic proton (6b). Methoxylated indoles could result in $98 \%$ and $78 \%$ of desired products respectively ( $\mathbf{7 b}$ and $\mathbf{8 b}$ ). Indole derivatives bearing the chloro, bromo and boryl groups resulted in good to excellent yields of products, which allow further cross coupling to achieve more complex settings (9b to $\mathbf{1 1 b}$ ). Unexpectedly, regiomers were isolated for substrates with an ester group (12b and $\left.\mathbf{1 2} \mathbf{b}^{\prime}\right)$. Then we moved the focus to a class of closely related $\mathrm{N}$ heterocycles, pyrroles. Pyrroles with phenyl, methyl, benzyl, dimethylamino and carbonato groups all gave around $70 \%$ of target difluoromethylthioethers (13b to $\mathbf{1 7 b})$. Of note is the substrate bearing a double bond, which is known to be reactive toward $\mathrm{PhSO}_{2} \mathrm{SCF}_{2} \mathrm{H}$. Gratifyingly, the $\mathrm{C}_{\mathrm{sp}^{2}}-\mathrm{H}$ difluoromethylthiolation product was selectively obtained (18b).

Other heteroarenes, e.g., azaindole, pyrazole, isoxazole, and chromone, which are pharmaceutically important scaffolds, were also effective (19b to $22 \mathbf{b}$ ). Notably, this protocol was viable for thiophene, which was generally unreactive in other difluoromethylthiolating recipes $(\mathbf{2 3 b}) \cdot{ }^{\mathbf{1 0 m , 2 0}}$ Finally, the versatility of this method on some arenes was explored. 1,3,5-Trimethoxybenzene was difluoromethylthiolated successfully (24b). As this method was proved adaptable to phenolic protons, installing $-\mathrm{SCF}_{2} \mathrm{H}$ on phenol (25b) and resorcinol derivatives (26b and $\mathbf{2 7 b}$ ) was efficient. Aniline and sulfide underwent difluoromethylthiolation smoothly as well, resulting in good yields (28b and $\mathbf{2 9 b}$ ).

Generally, high regioselectivity was observed for this difluoromethylthiolation reaction, which was a combined effect of electronics and sterics. In most cases, the site of the highest electron density was difluoromethylthiolated, e.g., C-2 in indoles and $\mathrm{C}-\mathbf{1}$ in pyrroles. When multiple sites of similar electron richness were available, the reaction occurred at the sterically less hindered $\mathrm{C}_{\mathrm{sp}^{2}}-\mathrm{H}$ (25b and 29b). Although an excess amount of $\mathrm{PhSO}_{2} \mathrm{SCF}_{2} \mathrm{H}$ was used, the difunctionalization product was rarely detected during our scope exploration (see $\mathrm{ESI} \dagger$ for details of mono-/difunctionalization and regioselectivity issues).

Knowing that the difluoromethylsulfoxides and difluoromethylsulfones ${ }^{28}$ are valuable entities in medicinal chemistry, we were encouraged to alter the oxidation state of sulfur in $-\mathrm{SCF}_{2} \mathrm{H}$. As expected, the oxidation of difluoromethylthioethers could be accomplished in a controlled manner under different oxidizing conditions (Scheme 4a). Besides, the versatility of this photochemical strategy was extended to produce other synthetically useful thiyl radicals. Under the optimal conditions, phenylthioether (30b) and naphthylthioether (31b) were furnished smoothly. However, alkylthiolation (32b) was undermined by the homocoupling event of alkylthiyl radicals. Moreover, 33a was subjected to a gram-scale experiment to demonstrate the practicality of this method (Scheme 4c). We were pleased to see that the reaction proceeded smoothly and a good yield of the desired product was obtained (33b).

Although the underlying mechanism remained obscure at this stage, our preliminary study hints at the radical nature of

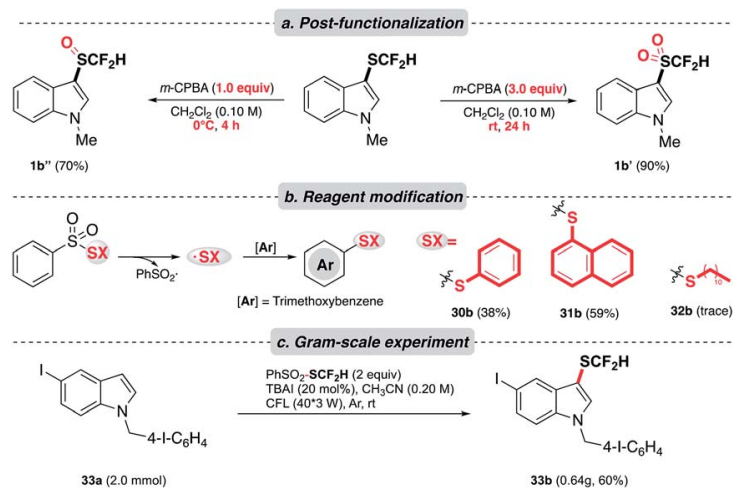

Scheme 4 Application. (a) Controlled oxidation of thioethers to corresponding sulfoxides and sulfones; (b) modified reagents to afford arylthiolation products; (c) scaled-up experiments.

this reaction as designed. The presence of various radical quenchers significantly impacted the desired reactivity (Scheme 5 , eqn (1) and (2)). When diallylmalonate (34a) was employed as the radical clock-type trapper, a cyclization adduct (34b) with $\mathrm{PhSO}_{2} \mathrm{SCF}_{2} \mathrm{H}$ was observed irrespective of the presence of $\mathbf{1 a}$ (eqn (2) and (3)). This result, to a degree, supports our postulation of the radical mechanism.

Based on the previous literature ${ }^{20,22 a}$ and the abovementioned experiments (see ESI $\dagger$ for details that rationalized the intermediacy of the proposed radical and the by-product formation), we envisioned that a radical-involved mechanism was operative. Under light irradiation, a difluoromethylthiyl radical resulted from either the homolysis of $\mathrm{PhSO}_{2} \mathrm{SCF}_{2} \mathrm{H}$ or the photo-induced electron transfer (PET) event between $\mathrm{PhSO}_{2} \mathrm{SCF}_{2} \mathrm{H}$ and iodide. Subsequently, the action of $\mathrm{SCF}_{2} \mathrm{H}$ radical on arenes was followed by hydrogen atom abstraction by phenylsulfonium (radical) to furnish the target compound. The resulting phenylsulfinic acid was unstable and further transformed into thiosulfonate. ${ }^{29}$

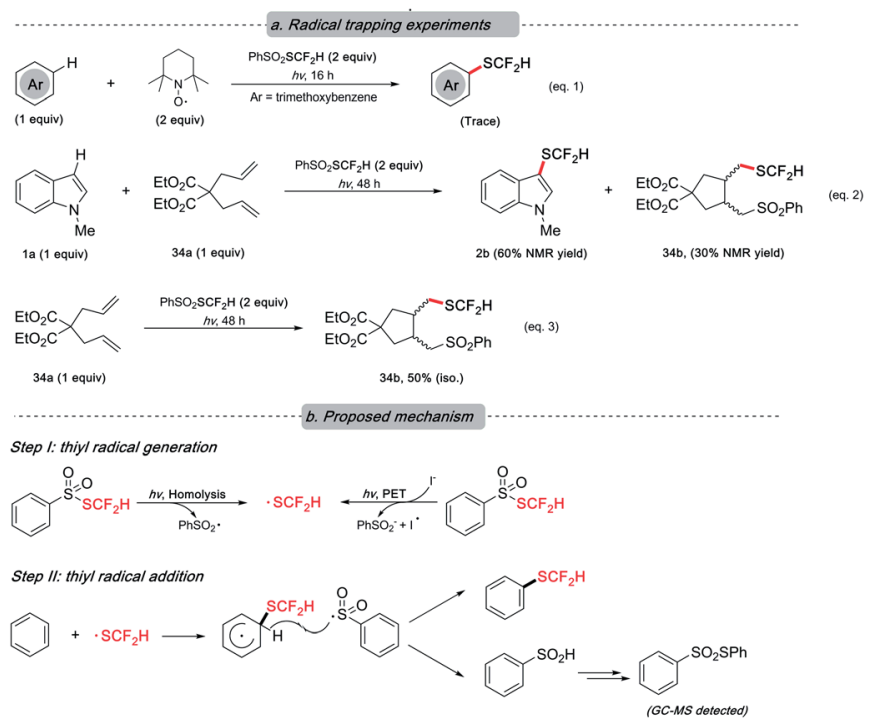

Scheme 5 Mechanistic study. (a) Termination of the desired reactivity in the presence of radical trappers; (b) plausible mechanism. 


\section{Conclusions}

In summary, we have developed a metal-catalyst-free aromatic difluoromethylthiolation reaction at room temperature enabled by visible light. This operationally simple strategy features the synthesis of a series of difluoromethylthioethers under mild conditions, which are a class of compounds with high medicinal value. ${ }^{1,2,3 b}$ These difluoromethylthioethers could be readily diversified into corresponding sulfones and sulfoxides. Moreover, this "dummy group" strategy holds great potential for achieving other types of radical thiolations by simply switching the functionalities tethered on thiosulfonate reagents. Details of mechanistic insight remain to be explored and we are dedicated to introducing fluorine-containing functional groups on arenes with similar strategies.

\section{Conflicts of interest}

There are no conflicts to declare.

\section{Acknowledgements}

We are grateful to the Canada Research Chair Foundation (for supporting C.-J. L.), the Canada Foundation for Innovation, the FQRNT Center in Green Chemistry and Catalysis, the Natural Sciences and Engineering Research Council of Canada, and McGill University for supporting our research. The authors also thank the Shanghai Institute of Organic Chemistry (SIOC) for supporting D. Zhu with a postdoctoral fellowship and supporting L. L with a National Postdoctoral Program for Innovative Talents fellowship (BX201700110). We are also grateful to Prof. Dr Dmitrii Perepichka (McGill University) for our access to the fluorescence facilities and Dr Robin Stein for her patience and helpful guidance on spectrometry.

\section{Notes and references}

1 (a) F. Leroux, P. Jeschke and M. Schlosser, Chem. Rev., 2005, 105, 827-856; (b) B. Manteau, S. Pazenok, J.-P. Vors and F. R. Leroux, J. Fluorine Chem., 2010, 131, 140-158; (c) C. Ni, M. Hu and J. Hu, Chem. Rev., 2014, 115, 765-825; (d) X.-H. Xu, K. Matsuzaki and N. Shibata, Chem. Rev., 2014, 115, 731-764.

2 (a) T. Fujita, J. Iwasa and C. Hansch, J. Am. Chem. Soc., 1964, 86, 5175-5180; (b) I. Rico and C. Wakselhan, Tetrahedron Lett., 1981, 22, 323-326.

3 (a) B. Bayarmagnai, C. Matheis, K. Jouvin and L. J. Goossen, Angew. Chem., Int. Ed., 2015, 54, 5753-5756; (b) Y. Zafrani, D. Yeffet, G. Sod-Moriah, A. Berliner, D. Amir, D. Marciano, E. Gershonov and S. Saphier, J. Med. Chem., 2017, 60, 797-804. 4 S. Okui, N. Kyomura, T. Fukuchi, K. Okano, L. He and A. Miyauchi, US Pat., 7371 768, 2008.

5 K. Morita, K. Ide, Y. Hayase, T. Takahashi and Y. Hayashi, Agric. Biol. Chem., 1987, 51, 1339-1343.

6 L. Yagupolskii, I. Maletina, K. Petko, D. Fedyuk, R. Handrock, S. Shavaran, B. Klebanov and S. Herzig, J. Fluorine Chem., 2001, 109, 87-94.
7 Z. Huang, O. Matsubara, S. Jia, E. Tokunaga and N. Shibata, Org. Lett., 2017, 19, 934-937.

8 H.-Y. Xiong, X. Pannecoucke and T. Besset, Chem.-Eur. J., 2016, 22, 16734-16749.

9 Selected examples for difluoromethylthiolation by reacting thiolates with " $\mathrm{CF}_{2}$ " species other than $\mathrm{CF}_{2}$ difluorocarbene: (a) W. Zhang, J. Zhu and J. Hu, Tetrahedron Lett., 2008, 49, 5006-5008; (b) G. S. Prakash, Z. Zhang, F. Wang, C. Ni and G. A. Olah, J. Fluorine Chem., 2011, 132, 792-798; (c) Y. Fujiwara, J. A. Dixon, R. A. Rodriguez, R. D. Baxter, D. D. Dixon, M. R. Collins, D. G. Blackmond and P. S. Baran, J. Am. Chem. Soc., 2012, 134, 1494-1497.

10 Selected examples for difluoromethylthiolation by reacting thiolates with : $\mathrm{CF}_{2}$ difluorocarbene: (a) B. R. Langlois, $J$. Fluorine Chem., 1988, 41, 247-261; (b) Q.-Y. Chen and S.-W. Wu, J. Fluorine Chem., 1989, 44, 433-440; (c) P. Deprez and J.-P. Vevert, J. Fluorine Chem., 1996, 80, 159162; (d) W. Zhang, F. Wang and J. Hu, Org. Lett., 2009, 11, 2109-2112; (e) Y. Zafrani, G. Sod-Moriah and Y. Segall, Tetrahedron, 2009, 65, 5278-5283; (f) F. Wang, W. Huang and J. Hu, Chin. J. Chem., 2011, 29, 2717-2721; $(g)$ L. Li, F. Wang, C. Ni and J. Hu, Angew. Chem., 2013, 125, 1261612620; (h) P. S. Fier and J. F. Hartwig, Angew. Chem., 2013, 125, 2146-2149; (i) C. S. Thomoson and W. R. Dolbier Jr, J. Org. Chem., 2013, 78, 8904-8908; (j) K. Fuchibe, M. Bando, R. Takayama and J. Ichikawa, J. Fluorine Chem., 2015, 171, 133-138; (k) J. Yu, J.-H. Lin and J.-C. Xiao, Angew. Chem., Int. Ed., 2017, 56, 16669-16673; (l) V. P. Mehta and M. F. Greaney, Org. Lett., 2013, 15, 5036-5039; (m) T. Ding, L. Jiang and W. Yi, Org. Lett., 2017, 20, 170-173.

11 Selected examples for difluoromethylthiolation by reacting disulfides with nucleophilic " $\mathrm{CF}_{2} \mathrm{H}$ " sources: (a) J. L. Howard, C. Schotten, S. T. Alston and D. L. Browne, Chem. Commun., 2016, 52, 8448-8451; (b) J.-B. Han, H.-L. Qin, S.-H. Ye, L. Zhu and C.-P. Zhang, J. Org. Chem., 2016, 81, 2506-2512; (c) Y.-m. Lin, W.-b. Yi, W.-z. Shen and G.-p. Lu, Org. Lett., 2016, 18, 592-595.

12 K. Jouvin, C. Matheis and L. J. Goossen, Chem.-Eur. J., 2015, 21, 14324-14327.

13 (a) J. Wu, Y. Gu, X. Leng and Q. Shen, Angew. Chem., Int. Ed., 2015, 54, 7648-7652; (b) J. Wu, Y. Liu, C. Lu and Q. Shen, Chem. Sci., 2016, 7, 3757-3762.

14 (a) D. Zhu, Y. Gu, L. Lu and Q. Shen, J. Am. Chem. Soc., 2015, 137, 10547-10553; (b) D. Zhu, X. Hong, D. Li, L. Lu and Q. Shen, Org. Process Res. Dev., 2017, 21, 1383-1387.

15 S. Arimori, O. Matsubara, M. Takada, N. Shibata and M. Shiro, R. Soc. Open Sci., 2016, 3, 160102-160110.

16 E. Ismalaj, D. Le Bars and T. Billard, Angew. Chem., Int. Ed., 2016, 55, 4790-4793.

17 H.-Y. Xiong, A. Bayle, X. Pannecoucke and T. Besset, Angew. Chem., Int. Ed., 2016, 55, 13490-13494.

18 F. Shen, P. Zhang, L. Lu and Q. Shen, Org. Lett., 2017, 19, 1032-1035.

19 (a) X. Zhao, A. Wei, T. Li, Z. Su, J. Chen and K. Lu, Org. Chem. Front., 2017, 4, 232-235; (b) Q. Yan, L. Q. Jiang, W. B. Yi, 
Q. R. Liu and W. Zhang, Adv. Synth. Catal., 2017, 359, 24712480.

20 D. Zhu, X. Shao, X. Hong, L. Lu and Q. Shen, Angew. Chem., Int. Ed., 2016, 55, 15807-15811.

21 B. Xu, D. Wang, Y. Hu and Q. Shen, Org. Chem. Front., 2018, 5, 1462-1465.

22 (a) S.-H. Guo, X.-L. Zhang, G.-F. Pan, X.-Q. Zhu, Y.-R. Gao and Y.-Q. Wang, Angew. Chem., Int. Ed., 2017, 57, 1663-1667; (b) S. H. Guo, M. Y. Wang, G. F. Pan, X. Q. Zhu, Y. R. Gao and Y.-Q. Wang, Adv. Synth. Catal., 2018, 360, 1861-1869; (c) B. Xu, D. Li, L. Lu, D.-C. Wang, Y. Hu and Q. Shen, Org. Chem. Front., 2018, DOI: 10.1039/c8qo00327k.

23 P. Liu, W. Liu and C.-J. Li, J. Am. Chem. Soc., 2017, 139, 14315-14321.

24 H. G. Viehe, Z. Janousek, R. Merenyi and L. Stella, Acc. Chem. Res., 1985, 18, 148-154.
25 (a) W. E. Lyons, Nature, 1948, 162, 1004; (b) O. Curcuruto, J. Winders, D. Franchi and M. Hamdan, Rapid Commun. Mass Spectrom., 1993, 7, 670-672.

26 F. Freeman and C. N. Angeletakis, J. Am. Chem. Soc., 1983, 105, 4039-4049.

27 (a) L. Li, W. Liu, H. Zeng, X. Mu, G. Cosa, Z. Mi and C. J. Li, J. Am. Chem. Soc., 2015, 137, 8328-8331; (b) L. Li, W. Liu, X. Mu, Z. Mi and C. J. Li, Nat. Protoc., 2016, 11, 1948-1954; (c) W. Liu, X. Yang, Y. Gao and C.-J. Li, J. Am. Chem. Soc., 2017, 139, 8621-8627.

28 (a) C. Rosinger, S. Shirakura, E. Hacker, Y. Sato, S. Heibges and S. Nakamura, Julius-Kühn-Archiv, 2012, 2, 544-548; (b) T. Yoshimura, T. Ikeuchi, S. Ohno, S. Asakura and Y. Hamada, J. Pestic. Sci., 2013, 38, 171-172.

29 C. J. M. Stirling, in The chemistry of sulphinic acids, esters and their derivatives, ed. S. Patai, John Wiley \& Sons, Chichester, New York, Brisbane, Toronto, Singapore, 1990, ch. 1, pp. 1-7. 